\title{
Radiotherapy Versus Cordectomy in the Management of Early Glottic Cancer
}

\section{Seung Yeun Chung, MD \\ Kyung Hwan Kim, MD \\ Ki Chang Keum, MD, PhD' \\ Yoon Woo Koh, MD, PhD² \\ Se-Heon Kim, MD, PhD² \\ Eun Chang Choi, MD, PhD² \\ Chang Geol Lee, MD, $\mathrm{PhD}^{1}$}

Departments of ${ }^{1}$ Radiation Oncology and ${ }^{2}$ Otorhinolaryngology, Yonsei Cancer Center, Yonsei University College of Medicine, Seoul, Korea

\begin{abstract}
Purpose
The purpose of this study was to compare the treatment outcomes of definitive radiotherapy
\end{abstract} (RT) with cordectomy in patients with early glottic cancer.

\section{Materials and Methods}

A total of 165 patients who were diagnosed with T1/2 squamous cell carcinoma of the glottic larynx between January 2006 and December 2012 were retrospectively analyzed. A total of 112 patients received RT and 53 patients received cordectomy. Local control (LC), diseasefree survival (DFS), overall survival (OS), and larynx preservation rates after RT and cordectomy were investigated.

\section{Results}

The median follow-up period was 77.7 months (range, 10.7 to 127.0 months). The 3 - and 5-year LC rates were $91.9 \%$ and $89.9 \%$, respectively, for the RT group, and $82.8 \%$ and $73.2 \%$, respectively, for the cordectomy group ( $p=0.006$ ). The 3- and 5-year DFS rates were $87.5 \%$ and $83.7 \%$, respectively, for the RT group and $79.2 \%$ and $68.0 \%$, respectively, for the cordectomy group ( $p=0.046$ ). No significant differences were identified in the 5-year OS $(92.8 \%$ vs. $90.6 \%, p=0.713)$ or larynx preservation rates $(98.2 \%$ vs. $97.2 \%, p=0.831)$ between groups. The major failure pattern was local failure $(n=26)$, followed by regional $(n=3)$ and distant failure $(n=2)$. Multivariate analysis of LC showed that T2 stage $(p=0.012)$ and receiving cordectomy as initial treatment $(p=0.001)$ were significantly associated with poorer LC.

\section{Conclusion}

RT resulted in higher rates of LC and DFS compared to cordectomy for early glottic cancer. Treatment with radiotherapy is feasible and should be encouraged for both T1 and T2 glottic cancer.

\author{
Correspondence: Chang Geol Lee, MD, PhD \\ Yonsei Cancer Center, Yonsei University \\ College of Medicine, 50 Yonsei-ro, \\ Seodaemun-gu, Seoul 03722, Korea \\ Tel: 82-2-2228-8114 \\ Fax: $82-2-2227-7823$ \\ E-mail: cglee1023@yuhs.ac \\ Received October 19, 2016 \\ Accepted March 10, 2017 \\ Published Online March 17, 2017
}

Key words

Laryngeal neoplasms, Radiotherapy, Local neoplasm recurrence

\section{Introduction}

Laryngeal cancer is one of the most common head and neck malignancies. It is estimated that approximately 13,560 new laryngeal cancer cases occurred in the United States in 2015, accounting for 3,640 deaths [1]. Among laryngeal cancers, the glottic larynx is the most common subsite, being involved approximately three times more often than the supraglottic larynx. Since glottic cancer involves the vocal cord, it is often diagnosed in the early stages owing to symptoms such as hoarseness. In early glottic cancer, lymph node metastasis is rare, with an incidence of clinically positive lymph nodes of nearly zero for T1 stage and $<2 \%$ for T2 stage, and a complete cure can often be achieved [2-4]. Therefore, the goal is to achieve the best local control (LC) leading to a complete cure and optimal functional results.

At present, there are various treatment modalities for treating early glottic cancer; namely, radiotherapy (RT), cordectomy, and surgery (e.g., partial laryngectomy). Although 
surgery has been used for decades in the past, its use has greatly decreased in recent years because of decreasing functional results and advances in RT and cordectomy [5].

The optimal treatment for early glottic cancer has continuously been an issue of debate, primarily because of the absence of results from large prospective randomized trials [6]. Recent studies have shown similar LC between RT and cordectomy. Mendenhall et al. [7] compared various nonrandomized data and reported LC rates ranging from approximately $80 \%$ to $94 \%$ for $\mathrm{T} 1$ tumors and $70 \%$ to $85 \%$ for T2 tumors for both modalities [8]. In such circumstances, decisions regarding the management of early glottic cancer vary across institutions and countries $[9,10]$. Since there are no ongoing randomized trials or expected randomized trials in the near future, it is important to review and compare retrospective studies. In our institution, all patients visited the Department of Otorhinolaryngology (ENT) first, and those who were determined to be not adequately resectable via cordectomy by ENT surgeons were referred to the Department of Radiation Oncology.

This study was conducted to evaluate LC, disease-free survival (DFS), overall survival (OS), and larynx preservation rates after RT or cordectomy as treatment options for early glottic cancer.

\section{Materials and Methods}

\section{Patients}

We retrospectively reviewed the medical records of patients who were diagnosed with early stage squamous cell carcinoma of the glottic larynx between January 2006 and December 2012 in two major clinical institutes. Early stage glottic cancer was defined as stage Tis (in situ), T1-2 without nodal metastases. Patients diagnosed with double primary cancer $(\mathrm{n}=35)$, and patients who received treatment prior to $\mathrm{RT}$ or cordectomy $(\mathrm{n}=194)$ or received both treatments $(\mathrm{n}=39)$ were excluded. The remaining 165 patients were retrospectively analyzed.

All patients were diagnosed by laryngeal microsurgery biopsy and clinically staged according to the American Joint Committee on Cancer seventh edition cancer staging.

\section{Treatment}

Patients who were considered not adequately resectable by cordectomy were referred for RT. Of the 165 patients, a total of 112 received definitive RT. The median dose for definitive RT was 65.25 Gy (range, 58.5 to 69.75 Gy). The most commonly used dose scheme was 64 Gy in 32 fractions for T1 stage and 66 Gy in 33 fractions for T2 stage glottic cancer patients. The fractional dose was $2 \mathrm{~Gy}(\mathrm{n}=54), 2.25 \mathrm{~Gy}$ $(n=56)$, or other $(n=2)$. Two patients grouped as other received ipsilateral vocal cord irradiation with a fractional dose of 1.8 Gy or 2.3 Gy via tomotherapy. Definitive RT was performed by conformal RT ( $\mathrm{n}=105)$ or tomotherapy $(\mathrm{n}=7)$. The gross tumor was delineated as gross tumor volume (GTV). The clinical target volume (CTV) was delineated including the whole larynx. Planning target volume (PTV) was defined as the CTV plus an adequate margin to encompass the possible setting uncertainties. For patients who received conformal RT, a traditional method was used; namely, a box field with the superior border at the top of the thyroid cartilage, the inferior border at the bottom of the cricoid cartilage (for T2, one tracheal ring below the cricoid cartilage), the anterior border at a $1 \mathrm{~cm}$ skin flash anteriorly, and the posterior border at the anterior edge of the vertebral body. No cone down was performed in 22 patients, and the RT field was reduced to the ipsilateral vocal cord in 20 patients. For the other 63 patients, the box field size was reduced by 1 to $2 \mathrm{~cm}$ depending on the patient. The median cone down dose was $46 \mathrm{~Gy}$ (range, 36 to $54 \mathrm{~Gy}$ ). For those who received tomotherapy, but not ipsilateral vocal cord irradiation, the initial field was the delineated PTV, after which cone down was performed to GTV plus margin after 56 Gy.

A total of 53 patients received cordectomy, which was performed by one of two modalities: transoral laser excision $(n=44)$ or transoral robotic surgery $(n=9)$. Cordectomy types were type I (subepithelial cordectomy, $n=9$ ), type II (subligamental cordectomy, $\mathrm{n}=10$ ), type III (transmulscular cordectomy, $\mathrm{n}=5$ ), type IV (total cordectomy, $\mathrm{n}=1$ ), type $\mathrm{V}$ (extended cordectomy, $n=1)$, and unknown $(n=27)$. The type of cordectomy was determined by the physician. No patients received chemotherapy during or after treatment in either the RT or the cordectomy group.

\section{Outcome evaluation}

Follow-up was performed regularly in both the Departments of ENT and Radiation Oncology on an outpatient clinic basis. The follow-up schedule was 1 month after completion of treatment and every 3 months for the first year, every 6 months for 5 years, and then annually thereafter. Events of recurrence, metastasis, and toxicity were recorded during follow-up visits. All outpatient clinic reports were retrospectively reviewed in detail to identify any toxicity events. 
Table 1. Patient and tumor characteristics

\begin{tabular}{|c|c|c|c|c|}
\hline Characteristic & $\begin{array}{c}\text { Total } \\
(n=165)\end{array}$ & $\begin{array}{l}\text { Definitive RT } \\
\quad(n=112)\end{array}$ & $\begin{array}{l}\text { Cordectomy } \\
\qquad(n=53)\end{array}$ & p-value \\
\hline Age, median (range, yr) & $63(37-87)$ & $64(37-87)$ & $64(39-83)$ & 0.260 \\
\hline \multicolumn{5}{|l|}{ Sex } \\
\hline Male & $152(92.1)$ & $104(92.9)$ & $48(90.6)$ & 0.610 \\
\hline Female & $13(7.9)$ & $8(7.1)$ & $5(9.4)$ & \\
\hline \multicolumn{5}{|l|}{ Clinical $\mathrm{T}$ stage } \\
\hline $\mathrm{T} 1 /$ is & $143(86.7)$ & $90(80.4)$ & $53(100)$ & 0.001 \\
\hline $\mathrm{T} 2$ & $22(13.3)$ & $22(19.6)$ & 0 & \\
\hline \multicolumn{5}{|l|}{ Tumor type } \\
\hline Superficial & $54(32.7)$ & $30(26.8)$ & $24(45.3)$ & 0.022 \\
\hline Exophytic & $89(53.9)$ & $69(61.6)$ & $20(37.7)$ & \\
\hline Ulcerative & $15(9.1)$ & $10(8.9)$ & $5(9.4)$ & \\
\hline Unknown & $7(4.2)$ & $3(2.7)$ & $4(7.5)$ & \\
\hline \multicolumn{5}{|l|}{ Histologic grade } \\
\hline NOS & $46(27.9)$ & $27(24.1)$ & $19(35.8)$ & 0.253 \\
\hline Well & $69(41.8)$ & $48(42.9)$ & $21(39.6)$ & \\
\hline Moderate & $46(27.9)$ & $33(29.5)$ & $13(24.5)$ & \\
\hline Poor & $4(2.4)$ & $4(3.6)$ & 0 & \\
\hline \multicolumn{5}{|l|}{ AC involvement } \\
\hline Not involved & $112(67.9)$ & $74(66.1)$ & $38(71.7)$ & 0.639 \\
\hline Involved & $52(31.5)$ & $37(33.0)$ & $15(28.3)$ & \\
\hline Unknown & $1(0.6)$ & $1(0.6)$ & 0 & \\
\hline \multicolumn{5}{|l|}{ Location } \\
\hline Both/AC involvement & $55(33.3)$ & $41(36.6)$ & $14(26.4)$ & 0.195 \\
\hline Unilateral & $110(66.7)$ & $71(63.4)$ & $39(73.6)$ & \\
\hline \multicolumn{5}{|l|}{ Smoking history } \\
\hline Yes & $107(64.8)$ & $72(64.3)$ & $35(66.0)$ & 0.826 \\
\hline No & $58(35.2)$ & $40(35.7)$ & $18(34.0)$ & \\
\hline
\end{tabular}

Values are presented as number (\%). RT, radiotherapy; is, in situ; NOS, not otherwise specified; AC, anterior commissure.

\section{Statistical analysis}

The primary endpoint was LC, and secondary endpoints included DFS, OS, and larynx preservation rates. Larynx preservation was defined as preservation of the larynx without undergoing total laryngectomy. Laryngectomy types for larynx preservation such as supracricoid laryngectomy or vertical partial laryngectomy were not considered failures of larynx preservation. The cumulative probability of LC, DFS, OS, and larynx preservation rates were calculated by the Kaplan-Meier method and compared by the log-rank test. Univariate and multivariate analyses of LC were performed using Cox regression. Multivariate analysis was performed using a backward elimination approach including all of the variables.

\section{Results}

\section{Patients and tumor characteristics}

Patient and tumor characteristics are shown in Table 1. The median age was the same for both groups, and patient characteristics such as age, sex, tumor type, histologic grade, anterior commissure involvement, location, and smoking history were well balanced between groups (all p >0.05). There was a significant difference between the definitive RT and cordectomy group in $\mathrm{T}$ stage $(\mathrm{p}=0.001)$. In the RT group, 90 patients $(80.4 \%)$ had $\mathrm{T} 1 /$ is and 22 patients $(19.6 \%)$ had T2 stage disease. However, all 53 patients $(100.0 \%)$ in the cordectomy group had T1/is. There were three stage Tis patients in the RT group and eight in the cordectomy group. Tumor type also differed significantly between groups $(p=0.022)$. While the dominant tumor type in the RT group was the exophytic type, 
A

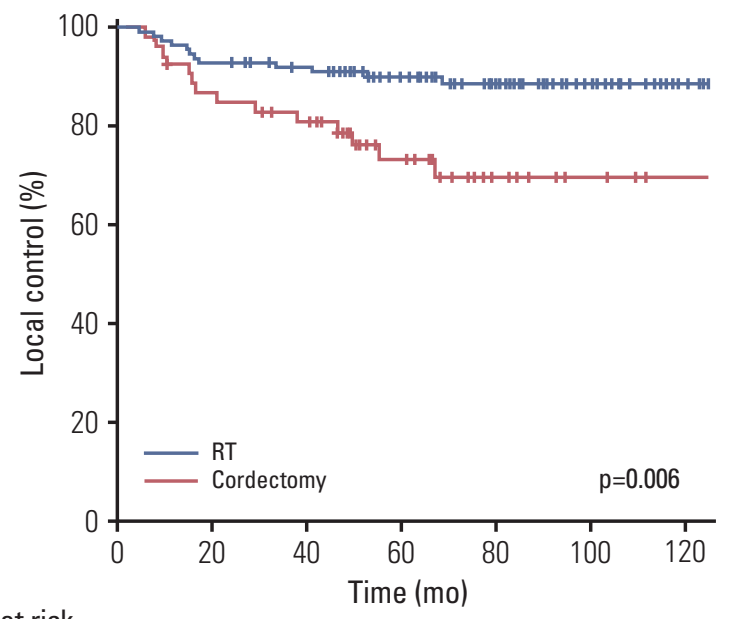

No. at risk

$\begin{array}{rrrrrrrr}\text { RT } & 112 & 104 & 97 & 78 & 58 & 34 & 12 \\ \text { Cordectomy } & 53 & 45 & 40 & 25 & 9 & 4 & 1\end{array}$

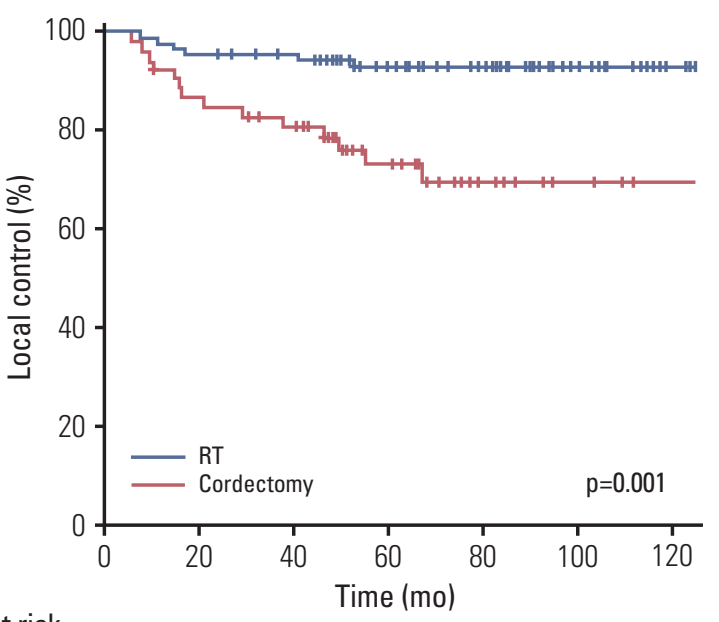

No. at risk

$\begin{array}{rrrrrrrr}\text { RT } & 90 & 86 & 81 & 64 & 51 & 29 & 10 \\ \text { Cordectomy } & 53 & 45 & 40 & 25 & 9 & 4 & 1\end{array}$

Fig. 1. Kaplan-Meier estimates of local control for $\mathrm{T} 1$ and $\mathrm{T} 2$ glottic cancer (A) and Kaplan-Meier estimates of local control for $\mathrm{T} 1$ glottic cancer (B). RT, radiotherapy.

Table 2. Multivariate Cox regression model for local recurrence

\begin{tabular}{|c|c|c|c|c|c|c|}
\hline \multirow{2}{*}{ Variable } & \multicolumn{3}{|c|}{ Univariate } & \multicolumn{3}{|c|}{ Multivariate } \\
\hline & HR & $95 \% \mathrm{CI}$ & p-value & HR & $95 \% \mathrm{CI}$ & p-value \\
\hline Age $(<65$ yr: $\geq 65$ yr $)$ & 1.45 & $0.67-3.14$ & 0.342 & - & - & - \\
\hline Sex (male:female) & 0.94 & $0.22-3.99$ & 0.936 & - & - & - \\
\hline T stage (T1/is:T2) & 2.05 & $0.82-5.11$ & 0.123 & 5.36 & $1.44-19.99$ & 0.012 \\
\hline Tumor type (superficial:exophytic/ulcerative) & 1.60 & $0.63-4.03$ & 0.320 & 3.39 & $0.95-12.13$ & 0.061 \\
\hline Histologic grade (WD:MD/PD) & 0.88 & $0.36-2.14$ & 0.769 & - & - & - \\
\hline AC involvement (no:yes) & 1.67 & $0.77-3.64$ & 0.197 & - & - & - \\
\hline Location (both/AC involvement:unilateral) & 0.66 & $0.30-1.43$ & 0.288 & - & - & - \\
\hline Smoking history (no:yes) & 1.27 & $0.55-2.91$ & 0.580 & - & - & - \\
\hline Treatment (radiotherapy:cordectomy) & 2.83 & $1.31-6.14$ & 0.008 & 7.79 & $2.35-25.87$ & 0.001 \\
\hline
\end{tabular}

$\mathrm{HR}$, hazard ratio; $\mathrm{CI}$, confidence interval; is, in situ; $\mathrm{WD}$, well differentiated; $\mathrm{MD}$, moderately differentiated; $\mathrm{PD}$, poorly differentiated; $\mathrm{AC}$, anterior commissure.

the dominant type in the cordectomy group was the superficial type. For the cordectomy group, a total of 26 out of 53 cordectomy cases reported the status of resection margin as positive or negative, and most did not report the length of the safety margin.

\section{Local control}

The median follow up period was 77.7 months (range, 10.7 to 127.0 months). LC was the primary endpoint. The 3- and 5 -year LC rates were $91.9 \%$ and $89.9 \%$ for the RT group and $82.8 \%$ and $73.2 \%$ for the cordectomy group, respectively ( $\mathrm{p}=0.006$ ) (Fig. 1A). Since no T2 patients received cordectomy, a subgroup analysis of only $\mathrm{T} 1$ glottic cancer patients was con- 


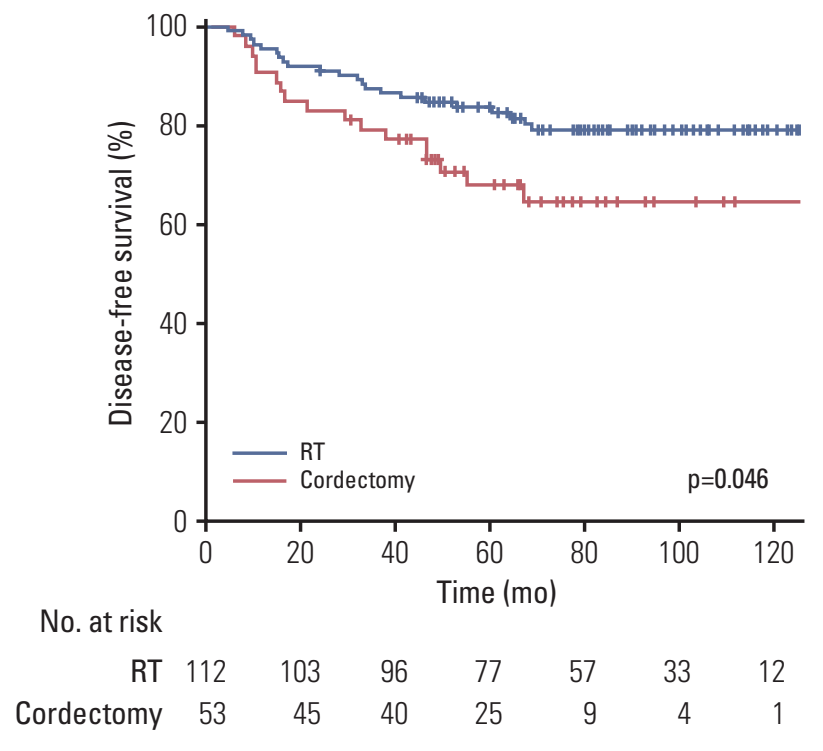

Fig. 2. Kaplan-Meier estimates of disease-free survival. $\mathrm{RT}$, radiotherapy.

ducted. For T1 patients ( $\mathrm{n}=143)$, the 3 - and 5 -year LC rates were $95.6 \%$ and $93.0 \%$, respectively, for the RT group $(\mathrm{n}=90)$, and $82.8 \%$ and $73.2 \%$ for the cordectomy group $(n=53)$ $(\mathrm{p}=0.001)$ (Fig. 1B). Multivariate analysis revealed that $\mathrm{LC}$ was adversely affected by a T stage of T2 (hazard ratio [HR], 5.36; 95\% confidence interval $[\mathrm{CI}], 1.44$ to $19.99 ; \mathrm{p}=0.012)$ and cordectomy as a treatment modality (HR, 7.79; $95 \% \mathrm{CI}, 2.35$ to 25.87; $\mathrm{p}=0.001$ ) (Table 2).

\section{Survival}

The 3- and 5-year DFS rates were $87.5 \%$ and $83.7 \%$, respectively, for the RT group and $79.2 \%$ and $68.0 \%$, respectively, for the cordectomy group ( $\mathrm{p}=0.046$ ) (Fig. 2). A subgroup analysis of T1 glottic cancer patients showed similar results with 3- and 5 -year DFS rates of $91.9 \%$ and $87.0 \%$, respectively, for the RT group and $75.5 \%$ and $64.8 \%$, respectively, for the cordectomy group ( $\mathrm{p}=0.007$ ). For OS, no significant differences were identified between the 3- and 5-year OS. The 3- and 5-year OS rates were $94.6 \%$ and $92.8 \%$, respectively, for the RT group and $94.3 \%$ and $90.6 \%$, respectively, for the cordectomy group $(\mathrm{p}=0.713)$ (Fig. 3).

Two patients in the RT group and one in the cordectomy group died of disease progression. Other causes of death were a newly developed second malignancy $(n=8)$, postoperative bleeding after salvage surgery $(\mathrm{n}=1)$, aspiration pneumonia $(n=1)$, and unknown because of follow-up loss with no evidence of disease (NED) status ( $\mathrm{n}=11$ ). The newly developed second malignancies were hepatocellular carcinoma, cholan-

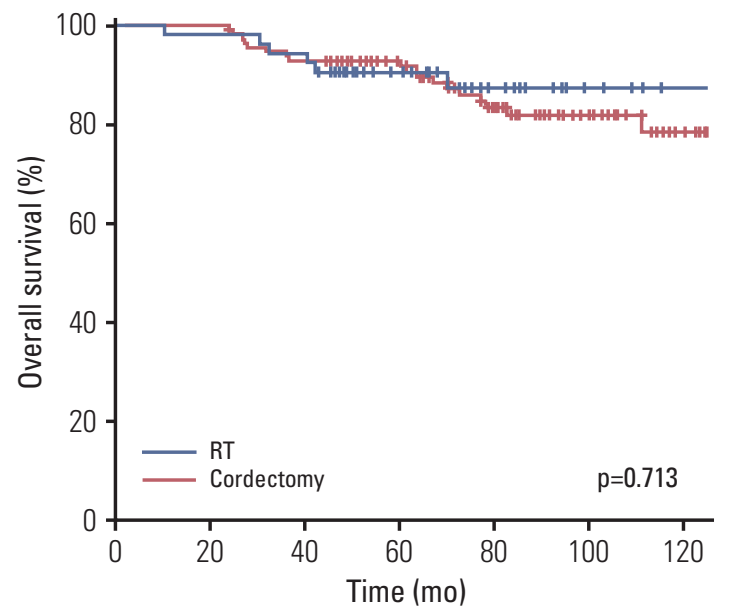

No. at risk

$\begin{array}{rrrrrrrr}\text { RT } & 112 & 111 & 103 & 86 & 62 & 36 & 12 \\ \text { Cordectomy } & 53 & 52 & 50 & 36 & 13 & 5 & 1\end{array}$

Fig. 3. Kaplan-Meier estimates of overall survival. RT, radiotherapy.

giocarcinoma, pancreatic cancer, esophageal cancer, and lung cancer.

\section{Patterns of failure and larynx preservation}

In the definitive RT group, a total of 12 local failures, three regional failures, and one distant failure occurred. All failures occurred independently. For local failures, failure sites included the involved vocal cord. For patients with recurrent stage $\mathrm{T} 1$ or T2N0M0, salvage treatment modalities were cordectomy $(n=4)$, re-irradiation $(n=1)$, supracricoid partial laryngectomy $(\mathrm{n}=1)$, and none because of a second primary cancer $(n=1)$. Among four patients who received salvage cordectomy, two were salvaged successfully, one died of lung cancer, and one died due to disease progression. For recurrent stage T3 or T4N0M0, salvage treatment modalities were total laryngectomy $(\mathrm{n}=3)$, supracricoid partial laryngectomy $(\mathrm{n}=1)$, and none because of follow-up loss $(n=1)$. Among regional failures, two were treated with salvage neck dissection followed by postoperative RT and one was treated with salvage chemoradiotherapy. One distant failure that occurred in the T-spine was successfully salvaged by RT.

In the cordectomy group, a total of 14 local failures and one distant failure occurred. Almost all local failure sites included the ipsilateral vocal cord, except for one failure that occurred in the contralateral vocal cord. One distant failure occurred as a hilar lymph node metastasis and was treated with RT. Salvage treatment modalities performed for local recurrences were laryngeal microsurgery $(\mathrm{n}=2)$, vertical partial laryngec- 
tomy or supracricoid partial laryngectomy $(\mathrm{n}=3)$, cordectomy $(n=3)$, RT ( $n=5)$, and none because of follow-up loss $(n=1)$. All five patients who received salvage RT were salvaged successfully.

Larynx preservation rates showed no significant difference between the groups. For the RT group, the 3- and 5-year larynx preservation rates were $98.2 \%$, while they were $100.0 \%$ and $97.2 \%$ in the cordectomy group $(\mathrm{p}=0.831)$.

\section{Complications}

During RT, three patients experienced a grade III skin reaction and two experienced grade III pharyngitis. Additionally, one patient required a 1-week break during treatment because of a grade III skin reaction. There were no late complications more severe than grade 2 . When follow-up of the patients in the RT group was conducted in the radiation oncology department, subjective scoring of the voice quality was performed. The patient scored the voice quality in a scale of 0 to 100 . The score for most patients was more than 80 , but six patients (5.4\%) still complained of continued hoarseness at a median of 10 months (range, 3 to 44 months) after treatment. In the cordectomy group, in one institution, patients who complained of severe voice impairment underwent assessment that included speech intelligibility and the percentage of consonants correct as criteria, which resulted in three patients being diagnosed with phonation disorder. In the other institution, voice handicap index was assessed for two-thirds of the patients who received cordectomy, and a total of six patients $(11.3 \%)$ complained of moderate to severe voice impairment at a median of 13 months (range, 2 to 46 months) after cordectomy.

\section{Discussion}

In our study, the RT group had better LC and DFS than the cordectomy group, with a 5-year LC rate of $89.9 \%$ and $73.2 \%$, and a DFS rate of $83.7 \%$ and $68.0 \%$, respectively. In addition, multivariate analysis revealed that LC was adversely affected by $\mathrm{T}$ stage and cordectomy as the first treatment choice. Currently, there are variable results regarding what the optimal treatment modality for early glottic cancer is, and to the best of our knowledge, this is the first study to show favorable results for RT.

In comparison to our results, previous studies have shown different results concerning LC. O'Hara et al. [11] conducted a review of 36 publications and concluded that there was no demonstrable difference in LC between RT and transoral laser surgery. The 3-year LC rates were $89.3 \%$ and $88.9 \%$ for
RT and transoral laser surgery, respectively, for stage IA tumors and $86.2 \%$ and $76.8 \%$, respectively, for stage IB tumors [11]. Another comprehensive systemic review by Yoo et al. [6] also concluded that there is no evidence in favor of either treatment.

An Italian study of RT alone showed 3-year and 5-year LC rates of $86 \%$ and $84 \%$, respectively, for $831 \mathrm{~T} 1$ glottic cancer cases. For $256 \mathrm{~T} 2$ glottic cancer patients, the 3-year and 5-year LC rates were both $73 \%[12,13]$. In our study, the results were superior for T1 stage, with 3-year and 5-year LC rates of $95.6 \%$ and $93 \%$, respectively, and 3-year and 5-year LC rates of $77.0 \%$ for both for stage T2. For cordectomy, Canis et al. [14] reported a 5-year LC rate of $86.8 \%$ for 404 patients with T1a glottic cancer who received transoral laser microsurgery. The results were inferior in our analysis, with a 5-year LC rate of $73.2 \%$ for $\mathrm{T} 1$ patients. Therefore, the favorable results for RT group in our study may have been due to the superior results of the RT group and the inferior results of the cordectomy group compared to previous reports.

In our study, the RT field was uniformly set for nearly all patients. The RT field generally not only included the gross tumor area but also areas concerned with microscopic extension. Cordectomy, on the other hand, has anatomical limitations that makes it difficult to expose the tumor adequately, especially with thyroid cartilage, cricothyroid membrane, and para-arytenoid musculature acting as limitations [15]. In addition, the extent of resection is always a dilemma for surgeons since resection extent is closely linked with voice quality [16]. Thus, cordectomy may not have been sufficient to control the local disease in some cases. While the results of cordectomy may vary across surgeons and institutions, when we examined whether there were any differences in LC between surgeons and the year in which cordectomy was performed, neither were found to differ significantly.

Although LC differed significantly between the RT and cordectomy groups, OS did not show any significant differences, which is consistent with the results of other studies [6]. A total of 26 local failures, three regional failures, and one distant failure occurred, but only three patients died of disease progression, demonstrating that recurrent glottic cancer could be salvaged in most cases in both treatment groups.

As for laryngeal preservation, there are various results reporting that there are no differences between the two treatments, or that the likelihood of laryngeal preservation may be higher with cordectomy [6-8]. Our results showed high rates of 5-year larynx preservation in both groups that did not differ significantly, with $98.2 \%$ and $97.2 \%$ being observed for the RT and cordectomy groups, respectively.

Since disease control rates of early glottic cancer are good with both treatment modalities, quality of voice is an essential concern. Aaltonen et al. [17] reported the first randomized study concerning voice quality of early laryngeal cancer 
patients. Patients who received RT or transoral laser surgery had similar overall voice quality, but RT resulted in a less breathy voice [17]. Others reported that voice quality was better in the RT group $[18,19]$. In our study, although there were limitations for the assessment of voice quality, there were more patients with severe voice impairments in the cordectomy group.

An interesting exception in the RT group was the two patients who received ipsilateral cord irradiation via tomotherapy, which is a modality attracting increasing interest with favorable results for both tumor control and promising voice quality [16]. These two patients have been followed up until now with NED status at 89.1 and 70.4 months, respectively. In addition, the fact that almost all local recurrences occurred at the ipsilateral vocal cord supports the possibility of treating only the ipsilateral vocal cord.

There are several limitations to our study, including its retrospective nature. In addition, objective voice quality assessment was not performed for all patients during follow-up. Thus, it is challenging to determine if one treatment modality is superior to another with respect to voice quality, which is an important factor in the decision about early glottic cancer treatment. Furthermore, the resection margins of specimens from cordectomy were not reported in many cases, making it difficult to confirm the cause of the lower LC in the cordectomy group.

However, to the best of our knowledge, this is the first study to demonstrate favorable results of RT over cordectomy. Currently, there are no prospective randomized trials and none are expected in the future; thus, decisions must be based on nonrandomized data. Most previous data show similar control rates of early glottic cancer for RT and cordectomy, and in some, a lower control rate for RT $[6,11,20]$. Since cordectomy outcomes can be influenced by many factors, including those associated with the location and surgeon, results may vary among studies.

In contrast to cordectomy, the favorable results of RT can be explained by unvarying inclusion of the tumor area in the RT field. Moreover, patients in the RT group did not experience any severe acute or late complications, and all but six patients $(5.4 \%)$ recovered voice quality. Thus, it seems reasonable that RT should be encouraged in both $\mathrm{T} 1$ and $\mathrm{T} 2$ glottic cancer, while cordectomy should be encouraged in adequately excisable cases of early glottic cancer.

RT resulted in higher rates of LC and DFS compared to cordectomy for early glottic cancer (T1/2), with low complication rates and acceptable voice quality. Treatment with radiotherapy is feasible and should be encouraged for both $\mathrm{T} 1$ and $\mathrm{T} 2$ glottic cancer.

\section{Conflicts of Interest}

Conflict of interest relevant to this article was not reported.

\section{References}

1. Siegel RL, Miller KD, Jemal A. Cancer statistics, 2015. CA Cancer J Clin. 2015;65:5-29.

2. Ries LA, Harkins D, Krapcho M, Mariotto A, Miller BA, Feuer EJ, et al. SEER cancer statistics review, 1975-2003. Bethesda, MD: National Cancer Institute; 2006.

3. Mendenhall WM, Amdur RJ, Morris CG, Hinerman RW. T1-T2N0 squamous cell carcinoma of the glottic larynx treated with radiation therapy. J Clin Oncol. 2001;19:4029-36.

4. Karatzanis AD, Psychogios G, Zenk J, Waldfahrer F, Hornung J, Velegrakis GA, et al. Comparison among different available surgical approaches in T1 glottic cancer. Laryngoscope. 2009; 119:1704-8.

5. Silver CE, Beitler JJ, Shaha AR, Rinaldo A, Ferlito A. Current trends in initial management of laryngeal cancer: the declining use of open surgery. Eur Arch Otorhinolaryngol. 2009;266: 1333-52.

6. Yoo J, Lacchetti C, Hammond JA, Gilbert RW; Head and Neck Cancer Disease Site Group. Role of endolaryngeal surgery (with or without laser) versus radiotherapy in the management of early (T1) glottic cancer: a systematic review. Head
Neck. 2014;36:1807-19.

7. Mendenhall WM, Werning JW, Hinerman RW, Amdur RJ, Villaret DB. Management of T1-T2 glottic carcinomas. Cancer. 2004;100:1786-92.

8. Abdurehim Y, Hua Z, Yasin Y, Xukurhan A, Imam I, Yuqin F. Transoral laser surgery versus radiotherapy: systematic review and meta-analysis for treatment options of T1a glottic cancer. Head Neck. 2012;34:23-33.

9. Makki FM, Williams B, Rajaraman M, Hart RD, Trites J, Brown $\mathrm{T}$, et al. Current practice patterns in the management of glottic cancer in Canada: results of a national survey. J Otolaryngol Head Neck Surg. 2011;40:205-10.

10. O'Sullivan B, Mackillop W, Gilbert R, Gaze M, Lundgren J, Atkinson $\mathrm{C}$, et al. Controversies in the management of laryngeal cancer: results of an international survey of patterns of care. Radiother Oncol. 1994;31:23-32.

11. O'Hara J, Markey A, Homer JJ. Transoral laser surgery versus radiotherapy for tumour stage $1 \mathrm{a}$ or $1 \mathrm{~b}$ glottic squamous cell carcinoma: systematic review of local control outcomes. J Laryngol Otol. 2013;127:732-8. 
12. Frata P, Cellai E, Magrini SM, Bonetti B, Vitali E, Tonoli S, et al. Radical radiotherapy for early glottic cancer: results in a series of 1087 patients from two Italian radiation oncology centers. II. The case of T2N0 disease. Int J Radiat Oncol Biol Phys. 2005;63:1387-94.

13. Cellai E, Frata P, Magrini SM, Paiar F, Barca R, Fondelli S, et al. Radical radiotherapy for early glottic cancer: results in a series of 1087 patients from two Italian radiation oncology centers. I. The case of T1N0 disease. Int J Radiat Oncol Biol Phys. 2005;63:1378-86.

14. Canis M, Ihler F, Martin A, Matthias C, Steiner W. Transoral laser microsurgery for T1a glottic cancer: review of 404 cases. Head Neck. 2015;37:889-95.

15. Davis RK, Jako GJ, Hyams VJ, Shapshay SM. The anatomic limitations of CO2 laser cordectomy. Laryngoscope. 1982; 92(9 Pt 1):980-4.

16. Mendelsohn AH, Xuan Y, Zhang Z. Voice outcomes following laser cordectomy for early glottic cancer: a physical model investigation. Laryngoscope. 2014;124:1882-6.

17. Aaltonen LM, Rautiainen N, Sellman J, Saarilahti K, Makitie A, Rihkanen $\mathrm{H}$, et al. Voice quality after treatment of early vocal cord cancer: a randomized trial comparing laser surgery with radiation therapy. Int J Radiat Oncol Biol Phys. 2014;90: 255-60.

18. Arias F, Arraras JI, Asin G, Uzcanga MI, Maravi E, Chicata V, et al. Quality of life and voice assessment in patients with early-stage glottic cancer. Head Neck. 2015;37:340-6.

19. Laoufi S, Mirghani H, Janot F, Hartl DM. Voice quality after treatment of T1a glottic cancer. Laryngoscope. 2014;124:1398401.

20. Brady JS, Marchiano E, Kam D, Baredes S, Eloy JA, Park RC. Survival impact of initial therapy in patients with T1-T2 glottic squamous cell carcinoma. Otolaryngol Head Neck Surg. 2016;155:257-64. 